\title{
Novel Carbon-Based Materials for Pressure and Force Sensors
}

\author{
Schmid-Engel, Hanna; Koppert, Ralf; Göttel, Dirk; Zohar, Keren; Berger, Shlomo and Schultes, Günter \\ University of Applied Sciences \\ Goebenstr. 40, D-66117 Saarbrücken, Germany
}

\section{Introduction}

The piezoresistive properties of amorphous carbon $(\mathrm{a}-\mathrm{C})$ and hydrogenated amorphous carbon $(\mathrm{a}-\mathrm{C}: \mathrm{H})$ were first discovered in the 90s of the last century. Until then these carbon materials were primarily applied as wear-resistant coatings due to their high mechanical hardness and chemical inertness.

In sensor applications based on the piezoresistive effect the change of electrical resistance caused by mechanical deformation due to pressure, strain etc. is measured by strain gauges. The sensitivity to strain of a piezoresistor is expressed quantitatively as the gauge factor (GF) which is defined as the ratio of the relative resistance change $\Delta R / R$ to the strain $\varepsilon$.

$$
\begin{aligned}
& G F=\frac{\Delta R}{R \cdot \varepsilon} \\
& \frac{\Delta R}{R}=\varepsilon \cdot(1+2 v)+\frac{\Delta \rho}{\rho}
\end{aligned}
$$

The relative resistance change $\Delta R / R$ due to applied stress is a function of geometry and resistivity ( $\rho)$ changes. The cross-sectional area of a bulk material reduces in proportion to the longitudinal strain by its Poisson's ratio, $v$, which for most materials ranges from 0.2 to 0.5 . Geometric effects alone provide a GF of approximately 1.4 to 2.0 . If the value for the gauge factor exceeds 2 , other physical effects concerning the material of the piezoresistor must be responsible for the enhancement.

However not only the sensitivity to a certain mechanical property is important for sensor applications, but also the temperature invariance of the electrical resistance. The temperature coefficient of electrical resistance (TCR), defined as

$$
T C R=\frac{\Delta R}{R \cdot \Delta T}
$$

should be ideally close to zero.

We study the piezoresistivity of metal-containing, especially nickel-containing hydrogenated amorphous carbon (Ni:a-C:H) thin films and explore the feasability of these novel carbon-based materials for sensor applications [1, 2]. The strain sensitivity of Ni:a-C:H thin films is enhanced by a factor of more than 10 compared to state of the art strain gauge materials and the TCR can be adjusted close to zero $(0 \pm$ $50 \mathrm{ppm} / \mathrm{K}$ ) by the Ni content. We focus on piezoresistive pressure sensors, which typically measure the deflection of a thin circular or rectangular membrane under an applied external pressure.

\section{Experimental}

$\mathrm{Ni}: \mathrm{a}-\mathrm{C}: \mathrm{H}$ films were prepared by a reactive sputtering process. In addition to the sputtering (RF power, $13.56 \mathrm{MHz}$ ) of a metallic target, a low percentage of a carbon containing precursor gas $\left(\right.$ e.g. $\left.\mathrm{C}_{2} \mathrm{H}_{4}\right)$ is added to the argon process gas. The metal is sputtered by the ionized argon depositing metal atoms on the substrate. Simultaneously, the precursor gas is decomposed by the plasma, building up a thin film of hydrogenated amorphous carbon on the substrate. As a result, metallic clusters are embedded within the hydrogenated carbon matrix. The base pressure of the recipient was approx. $1 \cdot 10^{-4} \mathrm{~Pa}$ and the pressure during the sputtering process was around 2.7 $\mathrm{Pa}$. As no heating during deposition is possible the samples were annealed in a load lock immediately before and after the deposition process. The Ni content was set by the $\mathrm{C}_{2} \mathrm{H}_{4}$ flow and the film thickness by the sputtering time.

Gauge factors were determined using a custom-made bending test setup built into a climate chamber by measuring the resistance change between a straight and a bent state (strain: $0.2 \%$ ). The TCR was determined by the resistance change due to a change in temperature without imposing any mechanical load. The samples used for the determination of the gauge factor and TCR were either $\mathrm{Al}_{2} \mathrm{O}_{3}$ substrates 
(Rubalit 710, CeramTec) or Si substrates with a $2 \mu \mathrm{m}$ thick insulating $\mathrm{SiO}_{2}$ layer (Siegert Consulting). The measurement structures were obtained by shadow masks or lift-off processes with an aluminum negative mask. The resistors have a U-structure with a line width of $1 \mathrm{~mm}$ and a total length of $10 \mathrm{~mm}$. The resistance was measured by a four-wire measuring method by probing the samples with four Au coated spring contacts.

\section{Results and discussion}

Fig. 1 shows that the temperature has a great influence on the strain sensitivity (gauge factor) of $\mathrm{Ni}: \mathrm{a}-\mathrm{C}: \mathrm{H}$ samples. This is especially the case for Ni contents of 50 to 65 at.\%, which is also the best composition to achieve a temperature independent electrical resistance (TCR of $0 \pm 50 \mathrm{ppm} / \mathrm{K}$ ). Gauge factors of up to 20 can be achieved for samples annealed at $300^{\circ} \mathrm{C}$ whereas samples that were annealed at $100{ }^{\circ} \mathrm{C}$ do not develop gauge factors higher than 14 . The conductivity of the films increases exponentially with increasing metal concentration. The percolation threshold is estimated to be at approx. 75 at. $\% \mathrm{Ni}$ content.

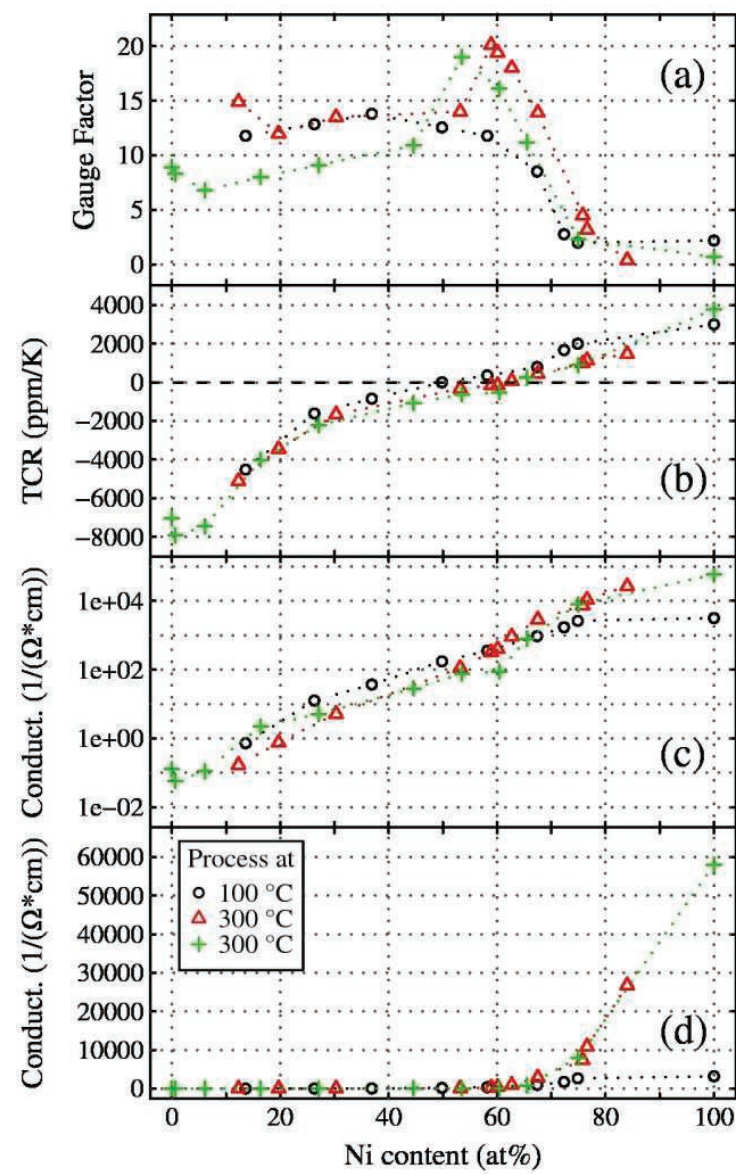

Fig. 1: Gauge factor (a), temperature coefficient of electrical resistance (b) and electrical conductivity in logarithmic (c) and linear scale (d) vs the metal concentration in Ni:a-C:H thin films. Data for films deposited on $\mathrm{Al}_{2} \mathrm{O}_{3}$ substrates at process temperatures of $100{ }^{\circ} \mathrm{C}$ (film thickness approx. $800 \mathrm{~nm}$ to $900 \mathrm{~nm}$ ) and $300{ }^{\circ} \mathrm{C}$ (film thickness approx. $300 \mathrm{~nm}$ to $400 \mathrm{~nm}$ ) respectively are presented.

Transmission electron microscopy (TEM) analysis of Ni:a-C:H cross sections prepared by focused ion beam (FIB) milling revealed a remarkable insight. Top view TEM images (reported elsewhere [3]) showed that the Ni:a-C:H films consist of nearly spherical Ni particles that are encapsulated by a few layers of graphite-like carbon. However, the cross sectional TEM images revealed a columnar growth of $\mathrm{Ni}$ particles perpendicular to the substrate surface. It has to be considered that the top view TEM samples were prepared with a thickness of approx. $70 \mathrm{~nm}$ to ensure a transparency to electrons whereas the cross sections were prepared from samples with $300 \mathrm{~nm}$ vertical thickness which is the standard thickness for the gauge factor samples. TEM images of the cross sections (see Fig. 2) show that the growth of elongated $\mathrm{Ni}$ particles starts from smaller spherical $\mathrm{Ni}$ particles which act as nucleation sites. With increasing sputtering time these spherical particles merge into elongated Ni particles, mostly densifying 
towards the surface of the films. The carbon surrounding the Ni columns is amorphous to graphite-like. The columnar growth is impeded if the sputtered $\mathrm{Ni}$ atoms are small in numbers compared to the carbon. In this case the carbon covers the $\mathrm{Ni}$ atoms before they are able to form larger elongated clusters.
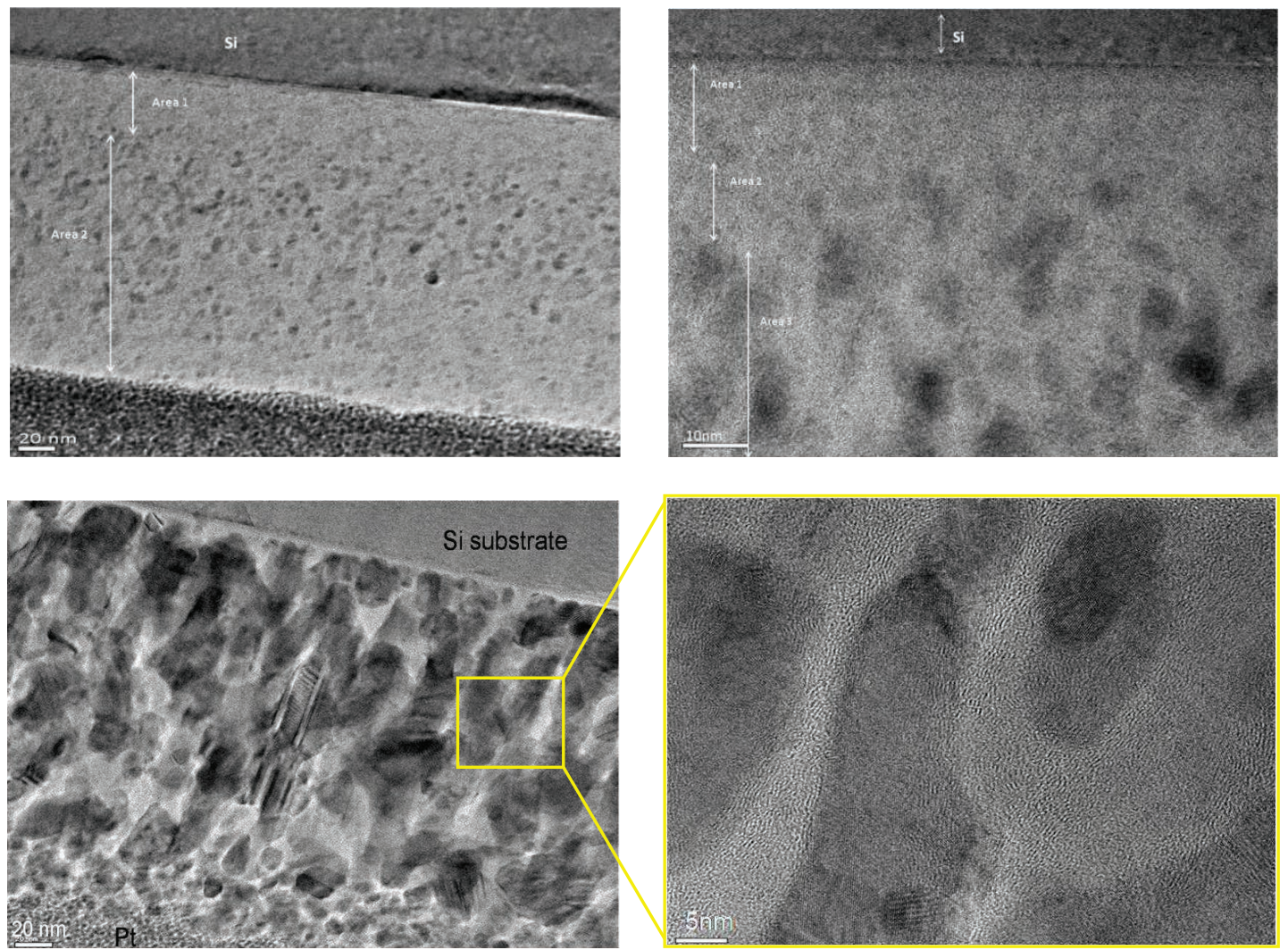

Fig. 2: TEM cross sections of Ni:a-C:H films with 4.5 at.\% Ni (top left), 12 at.\% Ni (top right) and approx. 55 at.\% $\mathrm{Ni}$ (bottom left and right). The bottom right picture which clearly shows the elongation of the Ni particles was taken from the same sample as the picture on the left, only with higher magnification. For samples with low Ni content a layer of amorphous carbon without any Ni clusters develops on the substrate (area1) prior to the growth of layers with $\mathrm{Ni}$ clusters (area 2 with nearly spherical clusters and area 3 with somewhat elongated particles respectively). Samples with high $\mathrm{Ni}$ content exhibit elongated Ni particles across the whole thickness except for the first 20-40 $\mathrm{nm}$ where smaller spherical particles are observed.

Field emission scanning electron microscopy (FE-SEM) top view images revealed another noteworthy phenomenon. The presence of some kind of superordinate unit (denominated as "superstructure" in this manuscript) was observed for samples with 50 to 60 at.\% Ni whereas this superstructure did not appear in samples with lower $\mathrm{Ni}$ content (see Fig. 3 and Fig. 4 respectively). The presence of a superstructure seems to be correlated with the presence of $\mathrm{Ni}$ columns grown vertically to the substrate. As these superstructures have diameters of 100 to $150 \mathrm{~nm}$ while each Ni column has a diameter of about 10 to $25 \mathrm{~nm}$ several columns must be involved in the formation of such superstructures.
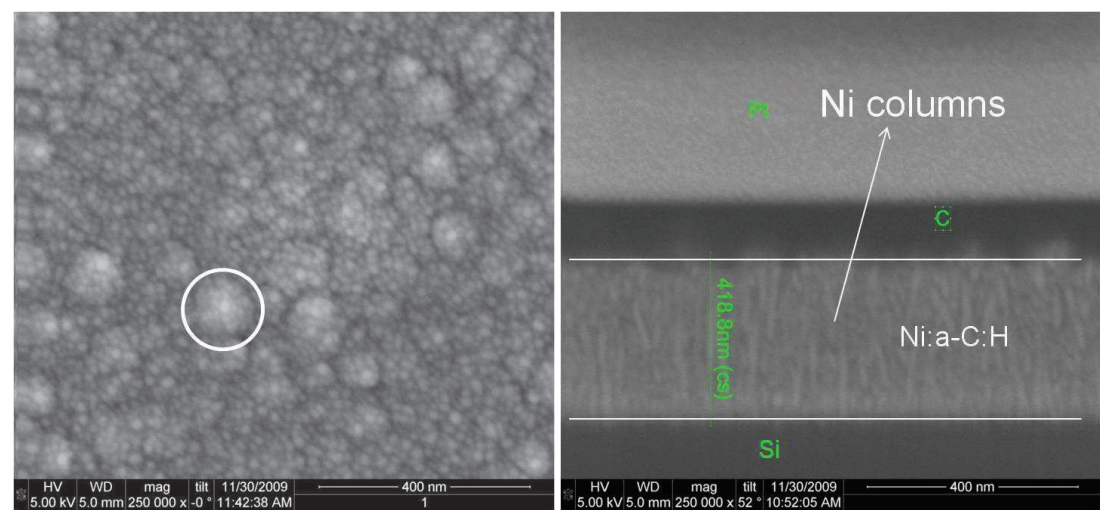
Fig. 3: FE-SEM top view (left) and cross-section (right) of a Ni:a-C:H sample with 53 at.\% Ni. Superstructures (one unit marked by circle) with unit sizes of 100 to $150 \mathrm{~nm}$ and Ni columns can be observed. The carbon layer on top of the Ni:a-C:H layer enhances the contrast. Platinum is deposited as protective layer to avoid damage during FIB milling.

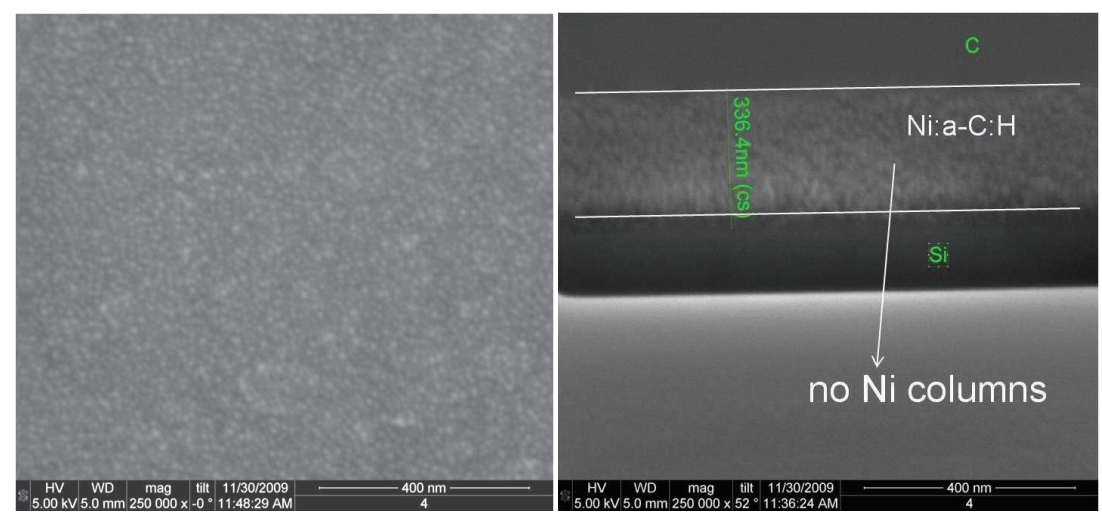

Fig. 4: FE-SEM top view (left) and cross-section (right) of a Ni:a-C:H sample with $\mathbf{3 0}$ at.\% Ni: no Ni columns and no superstructure can be observed.

The gauge factor does not depend on the film thickness as can be seen in Fig. 5. Therefore the vertical growth structure of the carbon-nickel nanocomposite seems to be irrelevant for the strain sensitivity. Only due to the roughness of the alumina substrates $\left(R_{a}: 0.06 \mu \mathrm{m} ; R_{\max }: 0.6 \mu \mathrm{m}\right)$ the gauge factor decreases with decreasing film thickness. This effect can be eliminated by depositing the films on silicon substrates $\left(R_{a}: 0.00 \mu \mathrm{m} ; R_{\max }: 0.05 \mu \mathrm{m}\right)$. By depositing the Ni:a-C:H films on substrates with excellent smoothness, gauge factor around 30 (30 \pm 2 ) can be achieved. The TCR is neither affected by the roughness of the substrate nor by the film thickness. It shows a more or less constant behavior over nearly the whole film thickness range except for films thinner than $80 \mathrm{~nm}$ where a strong decrease can be observed.
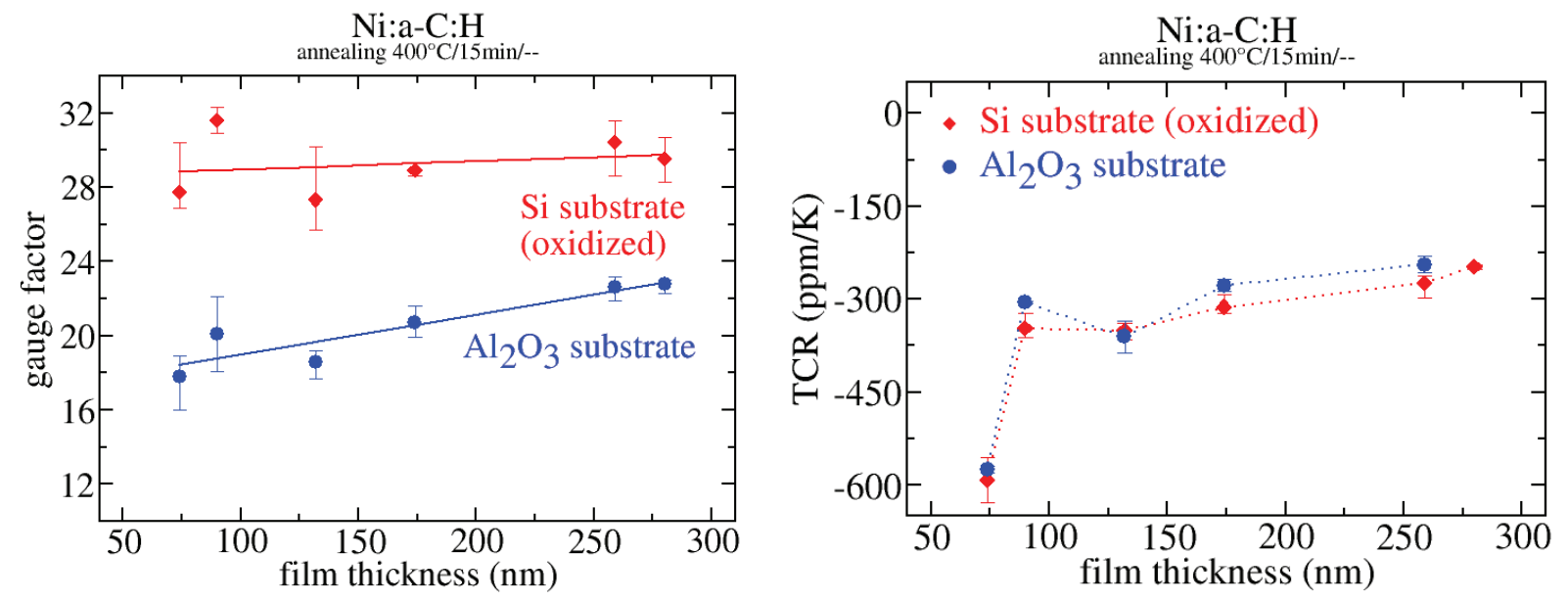

Fig. 5: Gauge factor and TCR of Ni:a-C:H films deposited on different substrates versus the film thickness.

To demonstrate the high application potential of these new functional layer a first series of steel based pressure sensors were accomplished. The functional layer consists of four resistors in the region of applied strain which are connected to a full Wheatstone brigde circuit. As a wet etching process is not possible for Ni:a-C:H thin films, the layout was attained by a lift-off process with an aluminum negative structure, so that a line width of around $50 \mu \mathrm{m}$ could be achieved. 


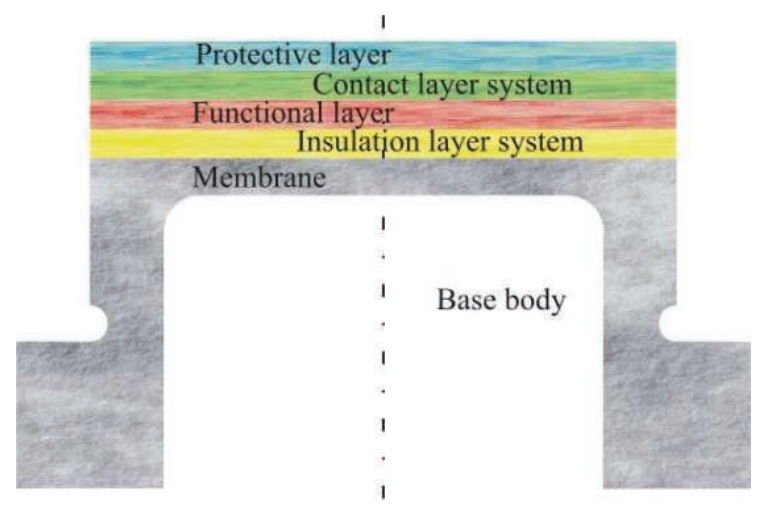

Fig. 6: Schematic cross-section of a steel based pressure sensor (membrane and thin film layers not true to scale)

The complete layer assembly of a typical steel based pressure sensor is shown in Fig. 6 . The thickness of the steel membrane is adjusted to the maximum pressure and is in the range of a few hundred microns. An insulation layer system consisting of $\mathrm{Al}_{2} \mathrm{O}_{3}$ and $\mathrm{Si}_{3} \mathrm{~N}_{4} / \mathrm{SiO}_{2}$ (overall thickness around $8 \mu \mathrm{m}$ ) separates the functional layer from the base body in order to reach breakdown voltages of up to $500 \mathrm{VDC}$. On top of the Ni:a-C:H functional layer a multilayer contact system is deposited consisting of a $\mathrm{CrNi}$ adhesion layer, a conduction layer of aluminum and a solderable layer of FeNi. As final layer a protective coating is applied to the sensor element to protect the thin film layers against environmental conditions like humidity. The offset was trimmed by an ESI laser system to approx. $\pm 50 \mu \mathrm{V} / \mathrm{V}$. To stabilize the electrical resistance the pressure sensors were annealed at different temperature levels up to $225^{\circ} \mathrm{C}$ for a few hours.

Measurements of the output signal of the new sensors based on Ni-C nanocomposites show a full-scale (FS) signal of approx. $20 \mathrm{mV} / \mathrm{V}$ compared to $2 \mathrm{mV} / \mathrm{V}$ of state of the art steel based pressure sensors with $\mathrm{NiCr}$ alloys as functional layer (see Fig. 7). The errors of linearity and hysteresis are below $0.4 \% \mathrm{FS}$ and $0.06 \% \mathrm{FS}$ respectively. These values are in good agreement with the standard pressure sensors with this kind of steel body. Hence, these errors are caused by the non-linear deformation of the steel membrane.

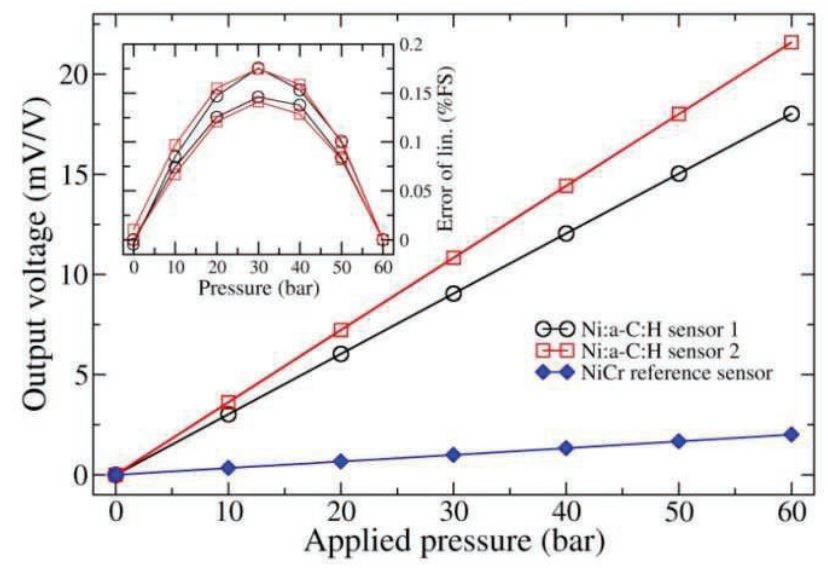

Fig. 7: Output signal and errors of linearity and hysteresis of two Ni:a-C:H pressure sensors compared to a state of the art pressure sensor based on a NiCr functional layer.

The sensitivity of membrane based pressure sensors can be increased by decreasing the thickness of the membrane. The controllable membrane thickness limits the pressure range, i.e. a thin and extended membrane is necessary to measure low pressures. However, the disadvantages of very thin membranes are non-linearities and difficulties in handling. As the output signal of the Ni:a-C:H pressure sensors is ten times higher than standard $\mathrm{NiCr}$ sensors, it is possible to measure low pressures without reducing the membrane thickness. Fig. 8 demonstrates the measurement of a pressure range up to 6 bar with sensors which are originally designed for a full load pressure of 60 bar. As depicted in Fig. 8, the maximum output voltage is approx. $2 \mathrm{mVN}$ with minimized errors of linearity and hysteresis (both below $0.05 \% \mathrm{FS}$ ). Thus, 
the fundamental advantages are the enhancement of the overload pressure by a factor of ten and the minimized errors.

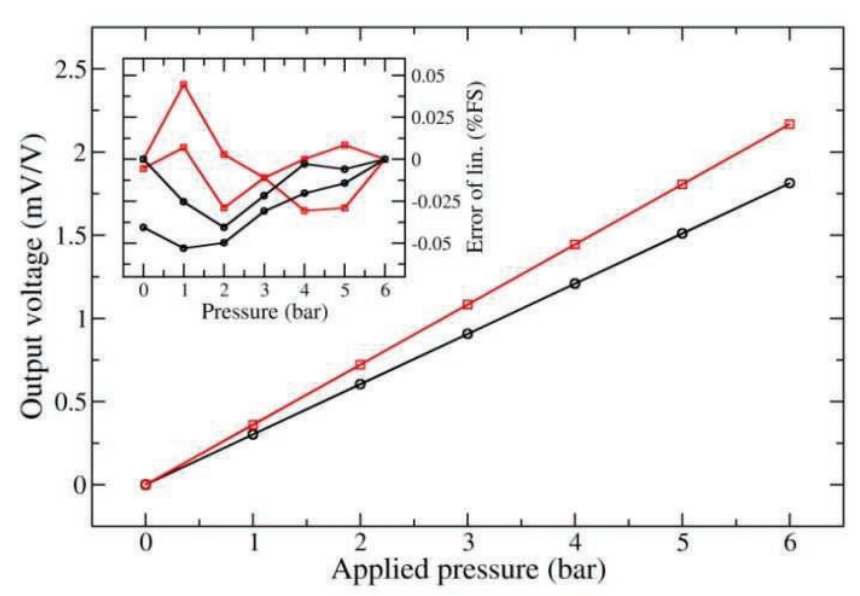

Fig. 8: Output signal of the same Ni:a-C:H pressure sensors as in Fig. 7 with a maximum load of 6 bar.

The temperature coefficient of sensitivity with a value of approx. $0.2 \% \mathrm{FS}$ is generally caused by the decrease of the modulus of elasticity with increasing temperature. The temperature coefficient of the offset is ten times higher. This high value could originate from a variation of the temperature coefficient of resistance of the four resistors of up to $\pm 25 \mathrm{ppm} / \mathrm{K}$.

Creep measurements reveal an average change of the output signal of approx. $-0.0225 \% \mathrm{FS}$ after 25 min at a pressure of 60 bar. The current noise of our new Ni:a-C:H functional layer was determined according to MIL-STD-202E in a direct comparison with high precision $\mathrm{NiCr}$ thin films on different substrates. Ni:a$\mathrm{C}: \mathrm{H}$ thin films exhibit current noises between $-20 \mathrm{~dB}$ and $-34 \mathrm{~dB}$ in contrast to constant values of $-35 \mathrm{~dB}$ for $\mathrm{NiCr}$ thin films. The large variation for the Ni:a-C:H thin films could be caused by unreliable adhesion properties of the contact system.

The temperature stability of the Ni:a-C:H thin films was analysed by a temperature hysteresis measurement. The offset of the pressure sensors was quantified after a temperature hysteresis between $-20^{\circ} \mathrm{C},+20^{\circ} \mathrm{C}$ and $+100^{\circ} \mathrm{C}$ with a load of $5 \mathrm{~V}$. After a holding time of $60 \mathrm{~min}$ at every temperature level and a threefold cycle the offset deviation of the samples tested is below $\pm 0.2 \% \mathrm{FS}$. Hence, most of the samples attain the stability criterion for this type of pressure sensors. First results of long term stability tests over $1000 \mathrm{~h}$ at a temperature of $125^{\circ} \mathrm{C}$ show a negligible offset deviation. Prior to the long term stability test a pre-stabilization process for $6 \mathrm{~h}$ at $200^{\circ} \mathrm{C}$ and $100 \mathrm{~h}$ at $150^{\circ} \mathrm{C}$ is conducted changing the offset of the Wheatstone bridge up to $1.5 \mathrm{mV} / \mathrm{V}$.

\section{Conclusions}

New perspectives for the electrical measurement of mechanical loads such as pressure, weight, force, etc. evolve from the investigation and the development of a class of metal containing hydrogenated carbon sensor films. The combination of high strain sensitivity and nearly temperature independent resistivity has been achieved. The process temperature is the crucial factor to achieve high strain sensitivities. The starting conditions (influenced by substrate roughness) of the film growth is important, however not the vertical film composition itself, as no correlation between the film thickness and the gauge factor has been observed. For the first time pressure sensors with Ni:a-C:H functional layers have been devised in order to demonstrate the high potential of this material. Their output signals (characteristic value of approx. $20 \mathrm{mV} / \mathrm{V}$ ) are ten times higher than those of state of the art pressure sensors, whereas the errors of linearity and hysteresis are comparable.

\section{References}

[1] G. Schultes, P. Frey, D. Göttel and O. Freitag-Weber, Diamond Rel. Mat. 15, 80 (2006)

[2] R. Koppert, D. Göttel, O. Freitag-Weber and G. Schultes, Solid State Sciences 11, 1797 (2009)

[3] R. Koppert, S. Uhlig, H. Schmid-Engel, D. Göttel, A-C. Probst, G. Schultes and U. Werner, submitted to J. Appl. Phys. (2011) 